\title{
Comportamiento productivo y características de la canal de conejos alimentados con harina de Moringa oleifera
}

\section{Productive behavior and carcass characteristics of rabbits fed with Moringa oleifera flour}

\section{Jerry Vivas Torres ${ }^{1}$, Nadir Reyes-Sánchez ${ }^{1}$, Alcides Sáenz ${ }^{1}$, Álvaro Benavides ${ }^{2}$}

'Facultad de Ciencia Animal / ORCID: https://orcid.org/0000-0001-9681-7215 / ORCID: https://orcid.org/0000-0002-5579-9396 /

${ }^{2}$ Facultad de Agronomía. Universidad Nacional Agraria.

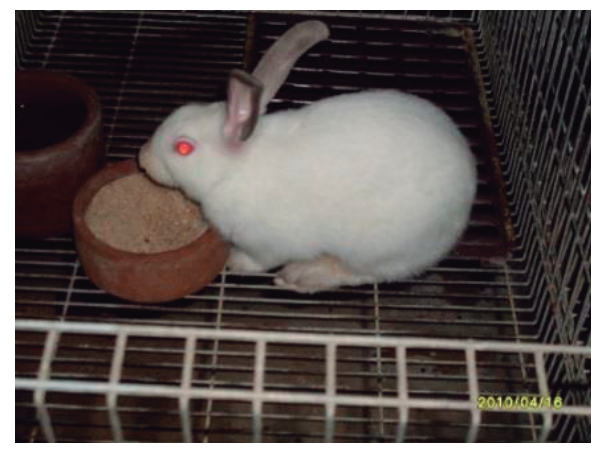

\section{RESUMEN}

Se utilizaron 36 conejos California y Nueva Zelanda Blanco con peso promedio de $554 \mathrm{~g}$ y 37 días de edad, en un diseño completamente aleatorizado con el objetivo de evaluar el efecto de la inclusión de Harina de Hojas Moringa oleifera (HHMO) en la alimentación de conejos, sobre el comportamiento productivo, características de la canal y morfometría del tracto gastrointestinal. Los tratamientos evaluados fueron T1: concentrado comercial, T2: concentrado con $16 \%$ de inclusión de HHMO y T3: concentrado con $19.64 \%$ de inclusión de HHMO. Se encontró que las mejores $(p<0,05) \mathrm{GMD}$ e ICA se obtienen con T1 (19.22 g/animal/día y 5.69) y T3 (20.49 g/ animal/día y 5.57), los que difieren estadísticamente $(p<0.05)$ del T2 (15.61 g/animal/día y 7.13). No se encontró efecto significativo de los tratamientos sobre el consumo de alimento. Con relación al peso vivo al sacrificio, peso canal caliente, rendimiento en canal, el peso del cuarto anterior, peso del lomo y peso del tórax de los conejos alimentados con CC fueron similares $(p>0.05)$ al de los conejos alimentados con $\mathrm{CC}$ con $19.64 \%$ de HHMO, pero significativamente mayores $(p<0.05)$ que el de los conejos alimentados con CC con $16 \%$ de HHMO. El peso del cuarto posterior, el peso del muslo y el ancho del lomo fueron significativamente mayores $(p<0.05)$ en los conejos alimentados con CC con $19.64 \%$ de HHMO. En la morfometría del TGI, no se encontró diferencias estadísticas entre los tratamientos. En conclusión, la inclusión del 19.64 \% de HHMO en el concentrado para conejos, no afecta el comportamiento productivo, ni las características de la canal, ni la morfometría del tracto gastrointestinal, siendo una alternativa viable para su utilización en granjas de pequeños productores.

Palabras clave: harina hojas Moringa oleifera, conejos, comportamiento productivo, morfometría.

\section{ABSTRACT}

36 California and New Zealand White rabbits with an average live weight of $554 \mathrm{~g}$ and 37 days of age were used in a completely randomized design with the objective to evaluate the effect of inclusion of Moringa oleifera leaf meal (MOLM) in rabbits feeding on the productive behavior, carcass characteristics and gastrointestinal tract morphometry. The treatments were: T1 commercial concentrate (CC); T2 CC with $16 \%$ of MOLM and T3 CC with $19.64 \%$ of MOLM. It was found that the best $(p<0.05)$ LWG and FCI were obtained with T1 (19.22 g/animal/day and 5.69) and T3 (20.49 g/ animal/day and 5.57), which differ statistically $(p<0.05)$ of T2 (15.61 g/animal/day and 7.13). For live weight at slaughter, carcass yield, loin and chest weight, no statistical differences $(p>0.05)$ were found between T1 and T3, but these differed significantly $(p<0.05)$ from T2. In conclusion, the inclusion of $19.64 \%$ of MOLM in the concentrate for rabbits, does not affect the productive behavior, the characteristics of the carcass and the morphometry of the gastrointestinal tract, being a viable alternative for its use in farms of small producers.

Keywords: Moringa oleifera leaf meal, rabbits, productive behavior, morphometry.
Recibido: 26 de julio del 2018 Aceptado: 31 de octubre del 2018

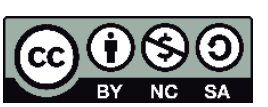

Los artículos de la revista La Calera de la Universidad Nacional Agraria, Nicaragua, se comparten bajo términos de la licencia Creative Commons: Reconocimiento, No Comercial, Compartir Igual. Las autorizaciones adicionales a las aquí delimitadas se pueden obtener en el correo freddy.aleman@ci.una.edu.ni 


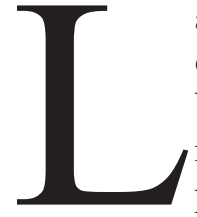

a cunicultura o crianza de conejos, se presenta como una alternativa para contribuir al combate del hambre y la desnutrición en áreas rurales, constituyendo una fuente idónea de proteína en la dieta de la población para el autoconsumo familiar, debido a que su carne tiene alto contenido nutricional que puede oscilar entre $14-20 \%$ de proteína, con bajos niveles de colesterol y ácidos grasos (Mesías et al. 2007). Esta actividad productiva es marginal con escasa tecnificación y realizada fundamentalmente por pequeños productores en el área rural. Los conejos son alimentados con forrajes y subproductos de la propia finca (Henríquez y Rizo 1994). Por otro lado, la utilización del componente arbóreo como recurso forrajero se considera una estrategia válida en los sistemas de producción sostenibles (Nieves 2005).

En la última década, el uso de follaje de árboles $\mathrm{y}$ arbustos forrajeros en forma de harina en dietas balanceadas destaca como una opción biológica y financieramente viable, por su excelente composición nutritiva, la capacidad de aprovechamiento digestivo de los conejos (Medugu et al., 2012) y la disponibilidad local del recurso arbóreo.

Moringa oleifera es un recurso arbóreo que crece bien en todo tipo de suelos desde ácidos hasta alcalinos (Duke, 1983), su producción de forraje varía entre 24 y 99 ton MS/ha/año, dependiendo del manejo agronómico (densidad de plantación, frecuencia de corte, fertilización), las hojas frescas contienen entre 17 y $27.4 \%$ de PB, 2.73 Mcal de EM/kg MS (Reyes-Sánchez et al., 2006; Reyes-Sánchez et al., 2006b; Reyes-Sánchez et al., 2009; Mendieta-Araica et al., 2011; Mendieta-Araica et al., 2013) y son ricas en vitaminas A, B y C, calcio, hierro y en aminoácidos esenciales.

La utilización de harina de follaje de árboles forrajero es una alternativa para la sustitución total o parcial de fuentes de proteínas convencionales (como la soya) en los alimentos balanceados para conejos, siendo un aspecto estratégico para la disminución de costos y la sostenibilidad biológica y financiera de la producción cunícola a nivel de pequeños productores

El objetivo del presente trabajo es evaluar la inclusión de harina de hoja de Moringa oleifera sobre el comportamiento productivo, características de la canal y morfometría del tracto gastrointestinal de conejos en desarrollo.

\section{MATERIALES Y MÉTODOS}

Ubicación del ensayo. El experimento se realizó en la granja experimental cunícola de la Facultad de Ciencia Animal (FACA) de la Universidad Nacional Agraria,
Managua, Nicaragua; localizada entre los $12^{\circ} 08^{\prime} 36^{\prime \prime}$ de la latitud norte y $86^{\circ} 09^{\prime} 48^{\prime \prime}$ de longitud oeste. Las condiciones climáticas en el sitio experimental corresponden a una zona de vida ecológica de bosque tropical seco, con un rango de precipitación histórica de $1132.4 \mathrm{~mm}$ anuales, humedad relativa del $71.5 \%$ y una temperatura media anual de $28.5^{\circ} \mathrm{C}$ (INETER 2010).

Animales experimentales. Se utilizaron 36 conejos mestizos de las razas California y Neozelandés blanco, con un peso vivo promedio de $554 \pm 73$ g y $37 \pm 3$ días de edad, los que fueron distribuidos en un Diseño Completamente Aleatorio (DCA) en tres tratamientos con 12 repeticiones por tratamiento. Los tratamientos en estudio fueron $\mathrm{T} 1$ : concentrado comercial (CC), T2: CC con inclusión de 16\% de HHMO y T3: CC con inclusión de 19.64\% de HHMO.

Instalaciones y equipos. Antes de iniciar el experimento se realizó la desinfección de la galera y las jaulas con creolina y formalina al $5 \%$, más flameo de las jaulas, encalado de las paredes y limpieza externa en las áreas adyacentes. Los conejos fueron alojados en jaulas metálicas dispuestas en sistema de batería tipo Flat-Deck (un solo piso), con su respectiva pila para deyecciones. Las dimensiones de cada jaula fueron $0.60 \mathrm{~m}$ de largo x $0.40 \mathrm{~m}$ de ancho x 0.34 $\mathrm{m}$ de alto, con bebederos de barrón y comedero de tolva metálico.

Manejo y alimentación de los animales. Los animales fueron sometidos a un período de adaptación (una semana) al régimen de alimentación y manejo. Posterior a la semana de adaptación, los animales se identificaron mediante tatuaje y fueron pesados individualmente, para proceder a su distribución aleatoria en los tres tratamientos.

A los 5 días de iniciado el experimento se les suministró $\mathrm{W}$ - plus coccidiostato a razón de $1.5 \mathrm{cc}$ por litro de agua como preventivo, repitiendo el suministro mensualmente, excepto en el mes que fueron sacrificados, para evitar residuos en la canal. Se les suministró vitamina $\mathrm{AD} 3 \mathrm{E}$ en el agua y en el transcurso del periodo experimental se suministró probiótico para estimular la flora microbiana del ciego y disminuir el estrés por el calor. En todos los tratamientos, la oferta de alimento por animal fue de $150 \mathrm{~g}$ dia-1, distribuidos tres veces al día (7:00 am. 12:00 m. y 5:00 pm) para evitar desperdicio y minimizar el estrés. El alimento ofrecido y el sobrante fue pesado y muestreado diariamente para posteriores análisis químicos. Se suministró agua ad libitum y se lavaron los bebederos cada 7 días, todas las actividades de manejo se realizaron a partir de las 7:00 am. 
Al concluir el período experimental (90 días), los conejos se sacrificaron utilizando el método tradicional de aturdimiento y degüello mediante corte de la vena yugular con un cuchillo afilado, para un desangrado completo, sin afectaciones en la calidad de la canal.

El desollado se llevó a cabo mediante la eliminación de la piel, incluida la cola y las patas, se abrió la carcasa, se realizó la extracción completa de las vísceras y se procedió al pesaje de la canal con y sin cabeza. A continuación, se identificó cada canal según tratamiento y repetición, se colocaron en bolsas plásticas y se enfriaron a temperaturas de 3 a $4^{\circ} \mathrm{C}$, posteriormente se descongelaron para proceder al despiece y estudio de las características de la canal realizar el despiece. Se pesaron (g) el cuarto anterior, el cuarto posterior, piernas, lomo, espadilla, tórax y muslo y se midió el ancho del lomo $(\mathrm{cm})$, utilizando una cinta métrica, siguiendo la metodología propuesta por Blasco et al., (1984).

\section{Preparación de la harina de hoja de Moringa oleifera} (HHMO). Se utilizó un área de M. oleifera con una densidad de 500000 plantas ha-1 $^{-1}$, manejada sin fertilización, sin herbicidas y sin riego. Antes de iniciar la elaboración de harina se realizó un corte de uniformidad, para garantizar la disponibilidad de rebrotes de 45 días de edad. Posteriormente, el follaje de Moringa se cortó con machete a una altura de $50 \mathrm{~cm}$ del suelo y se colocó sobre plástico de polietileno en capas de $10 \mathrm{~cm}$ de espesor con exposición plena a la luz solar para su secado, durante un período aproximado de 72 horas. Para garantizar un secado uniforme el follaje fue volteado cada 2 horas y se eliminaban tallos mayores a $5 \mathrm{~mm}$ de diámetro y los pecíolos. Una vez seco el material se procesó en un molino de martillo con una criba o tamiz de $3 \mathrm{~mm}$, obteniendo la HHMO para su posterior inclusión en las dietas experimentales.

Preparación de los tratamientos experimentales. Para la formulación del concentrado comercial (T1) se utiliza- ron los cuadros de requerimientos nutricionales para conejos según Lebas (2004), y se elaboró en la Planta Escuela de Alimentos Balanceados de la Universidad Agraria (PEAB-UNA).

El T2, se elaboró sustituyendo peso por peso (igual proporción) la harina de soya del concentrado comercial (T1) por HHMO (16\% de inclusión).

El T3, se formuló para que fuera iso-proteico e iso-energético respecto al $\mathrm{T} 1$, sustituyendo la harina de soya por HHMO (19.64\% de inclusión).

Análisis estadístico. Los datos fueron sometidos a análisis de varianza (ANDEVA) para determinar el efecto de los tratamientos sobre las variables estudiadas, usando el Modelo Lineal General (GLM) por medio del Software Statistical Analysis System (SAS) versión 9.1. Las comparaciones de medias se realizaron a través de la prueba de Duncan cuando las diferencias entre las medias fueron significativas $(p<0.05)$.

\section{RESULTADO Y DISCUSIÓN}

Ingredientes y composición química de los tratamientos experimentales. Los ingredientes y la composición química calculada de las raciones experimentales utilizadas en el estudio se muestran en los cuadros 1 y 2 .

Los requerimientos nutricionales de los conejos según Lebas, 2004 son: energía metabolizable (EM) 2700 $\mathrm{kcal} \mathrm{kg}^{-1}$; Proteína bruta (PB) entre 16 y $17 \%$ y Fibra bruta (FB) $14 \%$. Todas las raciones experimentales (T1, T2 y T3) utilizadas en el presente estudio cubren los requerimientos de PB, FB y EM, excepto en el caso de la PB ya que el T1 está por debajo de las necesidades nutricionales de los conejos (cuadro 2). Esto se debe a que el T2, se elaboró sustituyendo peso por peso (igual proporción) la harina de soya del CC por HHMO que tiene menor concentración de PB que la harina de soya. Es importante destacar que T3 fue formulado iso-energetico e iso-proteico con relación al T1 (cuadro 2).

Cuadro 1. Ingredientes utilizados en la formulación de las dietas experimentales

\begin{tabular}{|c|c|c|c|c|c|c|c|c|c|c|c|c|}
\hline \multicolumn{13}{|c|}{ Ingredientes } \\
\hline Tratamientos & $\begin{array}{l}\text { Sorgo } \\
\text { Rojo }\end{array}$ & $\begin{array}{l}\text { Semolina } \\
\text { de arroz }\end{array}$ & $\begin{array}{c}\text { Harina } \\
\text { de soya } \\
48 \%\end{array}$ & $\begin{array}{c}\text { Harina } \\
\text { hoja } \\
\text { Moringa }\end{array}$ & $\begin{array}{c}\text { Harina } \\
\text { carne } \\
\text { y hueso }\end{array}$ & $\begin{array}{c}\text { Cascarilla } \\
\text { mani }\end{array}$ & Melaza & $\mathrm{CaCo}_{3}$ & $\begin{array}{c}\text { Sal } \\
\text { común }\end{array}$ & $\begin{array}{c}\text { DL- } \\
\text { metioni } \\
\text { na }\end{array}$ & $\begin{array}{l}\text { Premix } \\
\text { broiler }\end{array}$ & Total \\
\hline $\mathrm{T} 1 \mathrm{CC}$ & 28.0 & 20.0 & 16.0 & - & 4 & 29.6 & - & 0.6 & 0.6 & 0.6 & 0.6 & 100 \\
\hline $\begin{array}{l}\text { T2 CC } \\
\text { con } 16 \% \text { HHMO }\end{array}$ & 28.0 & 20.0 & - & 16.0 & 4 & 29.6 & - & 0.6 & 0.6 & 0.6 & 0.6 & 100 \\
\hline
\end{tabular}


Cuadro 2. Composición química calculada de la formulación de las dietas experimentales

\begin{tabular}{|c|c|c|c|c|c|c|c|}
\hline Tratamientos & Humedad (\%) & MS (\%) & $\begin{array}{c}\text { Proteína } \\
(\mathrm{N} \times 6.25)\end{array}$ & Fibra $(\%)$ & Grasa (\%) & Ceniza $(\%)$ & $\begin{array}{c}\text { ED Calculada } \\
(\mathrm{kcal} / \mathrm{kg})\end{array}$ \\
\hline $\mathrm{T} 1 \mathrm{CC}$ & 11.48 & 88.52 & 17.70 & 14.92 & 3.19 & 6.00 & 2900 \\
\hline T2 CC con $16 \%$ HHMO & 11.63 & 88.37 & 13.91 & 15.78 & 3.44 & 6.52 & 2794 \\
\hline T3 CC con $19.64 \%$ HHMO & 11.15 & 88.85 & 17.49 & 15.32 & 4.01 & 6.39 & 2830 \\
\hline
\end{tabular}

T21: Isométrico respecto a T1: T31: Isoproteico respecto a T1. Fuente: Laboratorio LABAL (2010).

El aporte adecuado de FB de acuerdo a sus requerimientos (cuadro 2) permite a los conejos mejorar la digestibilidad de todos los componentes de la dieta y ayuda a mantener la salud intestinal al equilibrar la flora microbiana cecal y el tránsito digestivo (Dihigo, 2007).

\section{Efecto de la inclusión de HHMO en la alimentación} de conejos sobre el comportamiento productivo. Los resultados del comportamiento productivo de conejos de engorde alimentados con dietas experimentales que contienen harina de hoja de Moringa oleifera se muestran en el cuadro 3 . por Abubakar et al., 2015, Adeniji y Lawal 2012 y Nuhu 2010 que al alimentar conejos con niveles de inclusión de 5 a $30 \%$ de HHMO encontraron ganancias de pesos entre 7.7 y $15 \mathrm{~g}$ /día, estas diferencias pueden ser debido a que Abubakar et al., 2015 y Nuhu 2010, formularon los alimentos con menor contenido de FB entre 8.53 y $11.70 \%$.

Los valores obtenidos son similares a los reportados por Caro et al., 2013, entre 24.7 y 24.8 g/día en conejos alimentados con 15 y $30 \%$ de inclusión de HHMO en el CC. De igual manera, Dougnon et al., 2012, reportan 22.9 y 22.5 g día-1, al sustituir el CC por un 10 y $15 \%$ de Moringa peletizada, respectivamente.

Cuadro 3. Comportamiento productivo de conejos de alimentados con dietas experimentales que contienen harina de hoja de $M o$ ringa oleifera

\begin{tabular}{lccccc}
\hline \multicolumn{1}{c}{ Tratamientos } & PV inicial $(\mathrm{g})$ & PV final $(\mathrm{g})$ & GMD $(\mathrm{g})$ & Consumo $(\mathrm{g} / \mathrm{a} / \mathrm{d})$ & ICA \\
\hline T1 CC & 565.7 & $2295.5 \mathrm{a}$ & $19.22 \mathrm{a}$ & $109.3 \mathrm{a}$ & $5.69 \mathrm{a}$ \\
T2 CC con 16\% HHMO & 541.4 & $1946.3 \mathrm{~b}$ & $15.61 \mathrm{~b}$ & $111.33 \mathrm{a}$ & $7.13 \mathrm{~b}$ \\
T3 CC con 19.64\% HHMO & 590.7 & $2434.8 \mathrm{a}$ & $20.49 \mathrm{a}$ & $114.12 \mathrm{a}$ & $5.57 \mathrm{a}$ \\
\hline
\end{tabular}

Valores en una misma línea, seguidos de letras iguales no difieren estadísticamente entre sí, Duncan (P<0.05). PV: peso vivo; GMD: ganancia media diaria; CA: índice de conversión alimenticia.

Se observaron diferencias significativas $(p<0.05)$ entre los tratamientos experimentales estudiados (cuadro 4). Los resultados muestran que el mayor $(p<0.05)$ peso vivo final y ganancia media diaria de peso se obtuvieron en los conejos alimentados con T3 (2434.8 g y $20.49 \mathrm{~g})$ y T1 (2295.5 g y 19.22 g), los cuales no difieren significativamente entre sí. Los resultados obtenidos confirman lo expresado por García (2006), quien señaló que una ganancia media diaria de peso cercana a los $20 \mathrm{~g} /$ día se considera satisfactoria para climas tropicales.

Los pesos vivos finales de los animales se corresponden a los valores reportados (entre 2003 y 2236 g) por diversos autores (Sun et al., 2018; Etchu et al., 2017; Caro et al., 2013; Adeniji y Lawal, 2012; Dougnon et al., 2012) para esta etapa y superiores a los valores (entre 1150 y $1902 \mathrm{~g}$ ) reportados por Nuhu 2010 y Abubakar et al., 2015.

La ganancia media diaria de peso de los conejos alimentados con $\mathrm{T} 3$ son superiores a las reportadas
El efecto positivo de la inclusión de HHMO sobre el peso vivo final y la ganancia media diaria de peso en los conejos, se debe a que las hojas de $M$. oleifera contienen proteína dietética de excelente calidad (Odetola et al., 2012). Hay una mejora en la eficiencia de utilización de las proteínas en conejos alimentados con $M$. oleifera, debido a los altos contenidos proteicos y bajos niveles de fibra y lignina en el follaje de esta especie de planta (Abu Afsa et al., 2016)

Por otro lado, Nuhu (2010), expresa que la lisina y metionina son aminoácidos esenciales para el crecimiento óptimo de los conejos. La mayor ganancia de peso en los conejos alimentados con el T3 puede deberse, en parte, a una mejor calidad proteica, posiblemente derivada de un mayor suministro de metionina y lisina, producto de el excelente contenido de ambos aminoácidos en las hojas de M. oleifera (Makkar y Becker 1997). 
La menor ganancia diaria de peso (cuadro 2) observada en este estudio en los conejos alimentados T2 (15.61 g) podría explicarse por el hecho de que este tratamiento presentó el menor contenido de PB (13.91\%) comparado con los otros tratamientos (cuadro 1). El incremento en el contenido de PB en la dieta puede resultar en un aumento de peso diario, no obstante, debe coincidir con un aumento de aminoácidos como la metionina y la lisina, importantes para asegurar un mayor crecimiento.

El consumo diario de alimento no mostró diferencias significativas $(p>0.05)$ entre los tratamientos estudiados, sin embargo, los conejos mostraron un aumento sistemático en la ingesta diaria de alimento en los conejos que consumieron CC con inclusiones de HHMO (cuadro 2), lo que podría deberse a una mejor palatabilidad en las dietas con inclusiones de HHMO.

El consumo diario de alimento de los conejos que ingirieron CC con inclusión de HHMO (cuadro 2) son superiores a los valores de consumo (entre 60.1 y $103 \mathrm{~g} / \mathrm{d}$ ) reportados por Ghomsi et al., 2017; Abu-Hafsa et al., 2016; Adeniji y Lawal, 2012; Dougnon et al., 2012; Caro et al., 2013 y Nuhu 2010 para niveles de inclusión de entre 5 y 30\% de HHMO.

Según Caro et al., 2013 y García 2006, este comportamiento del consumo diario de las dietas con inclusión de HHMO pudo deberse a la voluminosidad de la HHMO, que impide un mayor consumo debido a factores físicos, es decir, a la capacidad del tracto digestivo (García 2006).

Sun et al., 2018, similar que, en el presente estudio, no obtuvo diferencias significativas en el consumo de alimento, sin embargo, encontraron valores de consumo superiores $(125.6,121.4$ y $125.3 \mathrm{~g} / \mathrm{d})$ al incluir niveles de 10,20 y $39 \%$ de HHMO, respectivamente.

El aumento del consumo de alimento en los conejos que ingieren dietas con inclusión de HHMO puede estar relacionado con la mejora en el perfil de aminoácidos, sobre todo de metionina y lisina, importantes para asegurar un mayor consumo (Forbes, 1995).

Las diferencias en resultados de consumo de alimento diario entre estas investigaciones pueden estar determinadas por factores referidos al animal o referidos al alimento que afectan el consumo de alimento como calidad y composición de la ración (contenido de proteína, contenido de fibra, contenido de energía, digestibilidad), forma de presentación del alimento, densidad y manejo, condiciones ambientales y de alojamiento, entre otros.

La conversión alimentaria estima la eficiencia con que se utiliza el alimento consumido para ganar una unidad de peso corporal. Se observaron diferencias significativas $(p<0.05)$ entre los tratamientos experimentales estudiados (cuadro 2). Los resultados muestran que el mejor $(p<0.05)$ ICA se obtuvo en los conejos alimentados con T3 (5.57) y con T1 (5.69), los cuales no difieren significativamente entre sí (cuadro 2). Los conejos que recibieron el T2 presentaron significativamente $(p<0.05 \%)$ el peor ICA (7.13), esto significa que consumieron $28 \%$ más de alimento que los conejos que ingirieron T3 para ganar un $\mathrm{kg}$ de peso corporal.

En la cuadro 3 se observa que los animales con una ganancia diaria de peso mayor (T1 y T3) muestran un ICA más eficiente $(p<0.05)$, probablemente debido a que no hay diferencias significativas en el consumo de alimento entre los tratamientos estudiados, de lo cual podemos inferir, que el similar consumo de alimento entre los tratamientos podría verse compensado con un mayor aprovechamiento digestivo de las dietas de T1 y T3.

El índice de conversión alimenticia obtenido en esta investigación con T3 (5.57) fue mejor que el valor de ICA (5.77) reportado por Adeniji y Lawal, 2012 para un nivel de inclusión de $20 \%$ de $\mathrm{HHMO}$ en la ración, pero fue peor que los valores de ICA (entre 3.16 y 4.63) reportados por otros autores (Sun et al., 2018; Ghomsi et al., 2017; Abu Hafsa et al., 2016; Caro et al., 2013; Dougnon et al., 2012; Djakalia et al., 2011 y Nuhu 2010) para niveles de inclusión de entre 10 y $30 \%$ de HHMO. Las diferencias en la constitución genética de los conejos utilizados para estos estudios pueden haber contribuido a las diferencias en valores de ICA registrados.

Efecto de la inclusión de HHMO en la alimentación de conejos sobre las características de la canal. En el cuadro 4 se muestran las características de la canal de los conejos alimentados con $\mathrm{CC}$ con diferentes niveles de inclusión de HHMO. El peso vivo al sacrificio, el peso de la canal caliente, el rendimiento de la canal, el peso del cuarto anterior, el peso del lomo y el peso del tórax de los conejos alimentados con T1 fueron similares $(p>0.05)$ al de los conejos alimentados con T3, pero significativamente mayores $(p<0.05)$ que el de los conejos alimentados con T2. El rendimiento en canal obtenido en el presente estudio en conejos alimentados con T1 y T3, se encuentra dentro del rango obtenido por otros autores (Cheeke, $1995 \mathrm{y}$ Roca, 2009) quienes reportan que el rendimiento en canal en conejos generalmente se encuentra entre el 50 y $65 \%$ del peso vivo del animal.

El peso del cuarto anterior del conejo según González (2010), representa el 13\% del peso de la canal. El valor obtenido en el peso del cuarto anterior de los conejos alimentados con T3, cae dentro del rango delimitado por este autor.

El peso del cuarto posterior, el peso del muslo y el ancho del lomo fueron significativamente mayores $(p<0.05)$ en los conejos alimentados con T3. 
Cuadro 4. Características de la canal de conejos de alimentados con dietas experimentales que contienen harina de hoja de Moringa oleifera

\begin{tabular}{|c|c|c|c|c|c|c|c|c|c|c|c|}
\hline Tratamientos & $\begin{array}{c}\text { PV al } \\
\text { Sacrificio } \\
(\mathrm{g})\end{array}$ & $\begin{array}{l}\text { Peso canal } \\
\text { caliente }(\mathrm{g})\end{array}$ & $\begin{array}{c}\text { Rendimiento } \\
\text { canal }(\%)\end{array}$ & $\begin{array}{l}\text { Cuadro } \\
\text { anterior } \\
(\mathrm{g})\end{array}$ & $\begin{array}{c}\text { Cuadro } \\
\text { posterior } \\
(\mathrm{g})\end{array}$ & $\begin{array}{l}\text { Peso } \\
\text { muslo } \\
(\mathrm{g})\end{array}$ & $\begin{array}{c}\text { Peso } \\
\text { lomo (g) }\end{array}$ & $\begin{array}{l}\text { Ancho } \\
\text { del lomo } \\
(\mathrm{cm})\end{array}$ & $\begin{array}{l}\text { Peso de } \\
\text { la pierna } \\
(\mathrm{g})\end{array}$ & $\begin{array}{c}\text { Peso de la } \\
\text { espaldilla (g) }\end{array}$ & $\begin{array}{l}\text { Peso del } \\
\text { torax }(\mathrm{g})\end{array}$ \\
\hline T1 CC & $2296 \mathrm{a}$ & $1265 \mathrm{a}$ & $55 \mathrm{a}$ & $180 \mathrm{a}$ & $358 \mathrm{~b}$ & $287 \mathrm{~b}$ & $261 \mathrm{a}$ & $5.5 \mathrm{~b}$ & $73.8 \mathrm{ab}$ & $97 \mathrm{ab}$ & $207 \mathrm{a}$ \\
\hline $\begin{array}{l}\text { T2 CC con } \\
16 \% \text { HHMO }\end{array}$ & $1946 \mathrm{~b}$ & $1007 \mathrm{~b}$ & $52 \mathrm{~b}$ & $147 \mathrm{~b}$ & $291 \mathrm{c}$ & $235 \mathrm{c}$ & $212 b$ & $5.3 \mathrm{~b}$ & $61.8 \mathrm{~b}$ & $77 \mathrm{~b}$ & $165 \mathrm{~b}$ \\
\hline $\begin{array}{l}\text { T3 CC con } \\
19.64 \% \\
\text { HHMO }\end{array}$ & $2435 \mathrm{a}$ & $1361 \mathrm{a}$ & $56 \mathrm{a}$ & $183 \mathrm{a}$ & $433 \mathrm{a}$ & $361 \mathrm{a}$ & $287 \mathrm{a}$ & $6.0 \mathrm{a}$ & $79.0 \mathrm{a}$ & $102 \mathrm{a}$ & $236 \mathrm{a}$ \\
\hline
\end{tabular}

Valores en una misma línea, seguidos de letras iguales no difieren estadísticamente entre sí, Duncan $(\mathrm{P}<0.05)$.

El cuarto posterior del conejo, anatómicamente es más desarrollado, por lo que contiene mayor proporción de carne comestible y generalmente es uno de los cortes valiosos preferidos por el consumidor. El mayor peso de este corte valioso en los conejos alimentados con T3 puede explicarse debido a que la sustitución parcial de la fuente de proteína del T1 por HHMO podría estar relacionado con la calidad nutricional de la HHMO que incluye su riqueza en minerales, vitaminas A, B, C y E y especialmente en proteínas de alto valor biológico con ocho aminoácidos esenciales (Odeyinka et al., 2008). Por su parte, Rweyemamu (2006) afirma que la combinación de HHMO y harina de soya puede mejorar el valor biológico de la proteína dietética en el sentido de mejorar la disponibilidad de aminoácidos limitantes, mejorando el rendimiento de los conejos.
El mayor peso del muslo $(p<0.05 \%)$ en los conejos alimentados con T3 puede ser explicado mediante lo reportado por Cobos (1993) en el sentido que la relación músculo/hueso de la región del muslo aumentó al mejorar el valor biológico de la proteína dietética.

El peso de la pierna y de la espaldilla fueron significativamente mayores $(p<0.05)$ en los conejos alimentados con T3, pero no difieren estadísticamente de los conejos alimentados con T1, los que a su vez no difieren del T2.

Efecto de la inclusión de HHMO en la alimentación de conejos sobre la morfometría del tracto gastrointestinal. Los niveles de inclusión de HHMO estudiados no tuvieron ningún efecto significativo $(p>0.05 \%)$ sobre la morfometría normal del tracto gastrointestinal de los conejos bajo estudio.

Cuadro 5. Morfometría del tracto gastrointestinal (peso promedio y desviación estándar) de conejos alimentados con dietas experimentales que contienen HHMO

\begin{tabular}{lccccc}
\hline \multicolumn{1}{c}{ Tratamientos } & $\begin{array}{c}\text { Estomago Lleno } \\
(\mathrm{g})\end{array}$ & $\begin{array}{c}\text { Contenido diges- } \\
\text { ta del estomago }\end{array}$ & $\begin{array}{c}\text { Ciego lleno } \\
(\mathrm{g})\end{array}$ & $\begin{array}{c}\text { Contenido diges- } \\
\text { ta del ciego }(\mathrm{g})\end{array}$ & $\begin{array}{c}\text { Longitud del } \\
\text { ciego }(\mathrm{m})\end{array}$ \\
\hline T1 CC & $102.9 \pm 23.2$ & $82.9 \pm 23.2$ & $107.9 \pm 24.5$ & $54.8 \pm 14.1$ & $0.46 \pm 0.05$ \\
colon recto $(\mathrm{m})$
\end{tabular}

Valores en una misma línea, seguidos de letras iguales no difieren estadísticamente entre sí, Duncan $(\mathrm{P}<0.05)$.

Cuadro 6. Morfometría del tracto gastrointestinal (peso promedio y desviación estándar) de conejos alimentados con dietas experimentales que contienen HHMO

\begin{tabular}{lcccc}
\hline \multicolumn{1}{c}{ Tratamientos } & Intestino delgado $(\mathrm{m})$ & Peso del hígado $(\mathrm{g})$ & Peso del mtiñon $(\mathrm{g})$ & Peso del corazón $(\mathrm{g})$ \\
\hline T1 CC & $2.59 \pm 0.05$ & 55.33 & 12.63 & 5.90 \\
T2 CC con 16\% HHMO & $2.52 \pm 0.06$ & 54.09 & 10.70 & 5.20 \\
T3 CC con 19.64\% & $2.56 \pm 0.11$ & 58.33 & 14.45 & 5.90 \\
HHMO & & & \\
\hline
\end{tabular}

Valores en una misma línea, seguidos de letras iguales no difieren estadísticamente entre sí, Duncan $(p<0.05)$. 


\section{CONCLUSIONES}

Los resultados de este trabajo sugieren que el empleo de un nivel de inclusión del 19.64\% de harina de hoja de Moringa oleifera en el concentrado comercial para conejos de engorde sustituyendo parcialmente la harina de soya no afecta su comportamiento productivo, ni las características de la canal, ni la morfometría del tracto gastrointestinal, siendo una alternativa viable para su utilización en granjas de pequeños productores.

\section{REFERENCIAS BIBLIOGRÁFICAS}

Abubakar, M; Ibrahim, U, Yusuf, AU, Muhammad, AS; Adamu, N. 2015. Growth performance, carcass and organ characteristics of growing rabbits fed graded levels of Moringa oleifera leaf meal in diets. Bayero Journal of Pure and Applied Sciences, 8(2):7-9. http://dx.doi.org/10.4314/bajopas.v8i2.2

Abu-Hafsa, SH; Salem, AZM; Hassan, AA; Kholif, AE; Elghandour, MMY; Barbabosa, A; Lopez, S. 2016. Digestion, growth performance and caecal fermentation in growing rabbits fed diets containing foliage of browse trees. World Rabbit Sci. 24: 283-293

Adeniji, AA; Lawal, M. 2012. Effects of replacing groundnut cake with Moringa oleifera leaf meal in the diets of grower rabbits. International Journal of Molecular Veterinary Research. 2:8-13.

Blasco, A; Estany, J; Baselga, M. 1984. Prediction of rabbit meat and bone weight using carcass measurements and simple cuts. Annales de zootecnie, INRA/EDP Sciences. 33(2):161-170. (en línea). Consultado 20 dic. 2013. Disponible en https://hal.archives-ouvertes. fr/hal-00888279/document

Caro, Y; Bustamante, D; Dihigo, LE, Ly, J. 2013. Harina de forraje de moringa (Moringa oleifera) como ingrediente en dietas para conejos de engorde. Revista Computadorizada de Producción Porcina 20(4):218-222.

Cheeke. P. 1995. Alimentación y nutrición del conejo. Acribia. Zaragoza, ES. 429 p.

Cobos, A. 1993. Influencia de la dieta en la composición lipídica en la carne de conejo. Tesis. Phd. Universidad Complutense de Madrid. Facultad de Veterinaria. Madriz, ES. 253 p. (en línea). Consultada 5 ago. 2011. Disponible en http://eprints.ucm.es/tesis/19911996/D/2/ AD2004901.pdf

Dihigo, L. 2007. Caracterización Físico-Química de productos tropicales y su impacto en la morfología digestiva del conejo. Tesis. Phd. Universidad Agraria de la Habana. 136 p. (en línea). Consultado 11 ago. 2011. Disponible en http://www.ica.edu.cu/biblioteca/Tesis/ dihigol.pdf

Djakalia, B, Louis, B; Soumaila, D. 2011. Effect of Moringa oleifera on growth permanence and health status of young post- waening rabbits. Research Journal of Poultry Sciences 4(1):7-13p (en línea). Consultado 16 jun. 2011. Disponible en http://docsdrive.com/ pdfs/medwelljournals/rjpscience/2011/7-13.pdf

Dougnon, TJ; Aboh, BA, Kpodékon, TM; Honvou, S; Youssao, I. 2012. Effects of substitution of pellet of Moringa oleifera to commercial feed on rabbit's digestion, growth performance and carcass trait. Journal of Applied Pharmaceutical Science 2(9):15-19.

Duke, JA. 1983. Handbook of energy crops (Moringa oleifera). Center for new crops and plant products. Purdue University, Indiana, US. http://www.hort.purdue. edu/newcrop/duke_energy/Moringa_oleifera.html

Etchu, AK; Tientcheu, BL, Ghomsi, MOS; Enow, JT, Tuedom, NM, Enamou, G. 2017. Effect of Moringa oleifera leaf meal (MOLM) on the growth, carcass, heamatology and biochemical parameters of rabbits. Vet Sci 3(3):1-5. DOI: 10.15226/2381-2907/3/3/00133.

Forbes, JM. 1995. Voluntary food intake and diet selection in farm animals. CAB International, Wallingford, U.K., pp. 7-8.

García, AM. 2006. Evaluación de forrajes tropicales en dietas para conejos de engorde. Tesis de Maestro en Ciencias en Industria Pecuaria. Universidad de Puerto Rico. Recinto Universitario de Mayagüez. 89 p.

Ghomsi, MOS; Enow, JT; Etchu, KA; Tientcheu, BL; Enamou, G; Chouengouong, TM; Mongo, BG; Bayemi, PH. 2017. Effect of Moringa oleifera leaf meal (MOLM) on the growth, carcass, heamatology and biochemical parameters of rabbits. SOJ Veterinary Sciences 3(3):1-5. DOI:10.15226/2381-2907/3/3/00133

González, P. 2010. La Producción de carne en Andalucía: La producción de carne de conejo en Andalucía. (en línea). Consultado 9 jul. 2011. Disponible en http://www.juntadeandalucia.es/opencms/opencms/system/bodies/contenidos/publicaciones/pubcap/2010/ pubcap_3445/La_produccixn_de_carne_en_Andalucxa.pdf

Henríquez, F; Rizo, J. 1994. Efecto de tres presentaciones de pienso sobre la productividad en conejos de engorde. Tesis. Ing. Agr. Universidad Nacional Agraria, Facultad de Ciencia Animal. Managua, NI. 55 p.

INETER (Instituto Nicaragüense de Estudios Territoriales). 2010. Registra estadísticos. Estación meteorológica del Aeropuerto Internacional Augusto Cesar Sandino. Las Mercedes. Managua, NI.

Lebas, F. 2004. Reflections on rabbit nutrition with a special emphasis on feed ingredients utilization: 8th World Rabbit Congress. Puebla, MX. 686 -736 p. (en línea). Consultado 25 abr. 2012. Disponible en http://cuniculture.info/Docs/Documentation/PubliLebas/2000-2009/2004-Lebas-WRC-Revue-sources-matiere-premieres-Puebla.pdf 
Makkar, HPS; Becker, K. 1997. Nutrients and antiquality factors in different morphological parts of the Moringa oleifera tree. Journal of agricultural Science, Cambridge 128: 311-332.

Medugu, CI; Mohammed, G; Raji, AO; Barwa, E; Andi-Zhinma, A. 2012. Utilization of different forages by growing rabbits. International Journal of Advanced Biological Research 2:375-381

Mendieta, B. 2011. Moringa oleifera as an alternative fodder for dairy cows in Nicaragua. (en línea). Thesis. Phd. University of Agricultural Sciences. Faculty of Veterinary Medicine and Animal Science. Uppsala. SE. Consultado 18 mayo 2011. Disponible en http://redMarango.una.edu.ni/documentos/Thesis-Moringa-with-papers-2011.pdf

Mendieta-Araica, B; Sporndly, E; Reyes-Sánchez, N; Salmerón-Miranda, F; Halling, M. 2013. Biomass production and chemical composition of Moringa oleifera under different planting densities and levels of nitrogen fertilization. Agroforestry Systems 87:8192.

Mesías, L; Parra, A; Ramírez, D; Orrego, G; Sarmiento, G. 2007. Diseño e implementación de un modelo técnico administrativo para la conformación de la mini cadena productiva cunícola dentro de la cadena de cárnicos en Santander estudio de demanda potencial. (en línea). Consultado 20 ago. 2011. Disponible en http://drupal.cvudes.edu.co/files/OI_161.doc

Nieves. D. 2005. VIII Encuentro de nutrición y producción de animales monogástricos: Forrajes promisorios para la alimentación de conejos en Venezuela, valor nutricional. (en línea). Consultado 25 mar. 2012. Disponible en http://www.avpa.ula.ve/eventos/ viii_encuentro_monogastricos/curso_alimentacion_no_convencional/conferencia-2.pdf

Nuhu, F. 2010. Effect of Moringa leaf meal (MOLM) on nutrient digestibility, growth, carcass and blood indices of weaner rabbits. Tesis. MSc. Kwame Nkrumah University of Science and Technoly, Faculty of Agriculture and Natural Resources, Department of Animal Science, Kumasi, GH. 122p. (en línea). Consultado 3 mar. 2011. Disponible en http://dspace.knust.edu.gh:8080/jspui/ bitstream/123456789/337/Binder1.pdf?

Odetola, OM; Adetola, OO; Ijaduola, TI; Adedeji, OY; Adu, OA. 2012. Utilization of Moringa oleifera leaves meal as a replacement for soya bean meal in rabbit's diets. Sch. J. Agri. Sci. 2(12):309-313.

Odeyinka, SM; Oyedele, OJ; Adeleke, TO; Odedire, JA. 2008. Reproductive performance of rabbits fed Moringa oleifera as a replacement for Centrosema pubescens, 9th World Rabbit Congress, Verona-Italy, p 411-415.

Reyes-Sánchez N; Ledin, S; Ledin, I. 2006. Biomass production and chemical composition of Moringa oleifera under different management regimes in Nicaragua. Agroforestry Systems 66:231-242

Reyes-Sánchez, N; Sporndly, E; Ledin, I. 2006 b. Effect of feeding different levels of foliage of Moringa oleifera to creole dairy cows on intake, digestibility, milk production and composition. Livestock Science 101:24-31

Reyes-Sánchez, N; Rodríguez, R; Mendieta-Araica, B; Mejía-Sovalbarro, L; Mora-Taylor, A. 2009. Efecto de la suplementación con Moringa oleífera sobre el comportamiento productivo de ovinos alimentados con una dieta basal de pasto guinea (Panicum maximun Jacq.). La Calera 9(13):60-69. Disponible en línea: http://www.lamjol.info/index.php/CALERA/article/view/19/18

Roca, T. 2009. Caracterización de la carne de conejo: Rendimiento en canal. (en línea). Consultado 6 jun. 2011. Disponible en http:// www.conejos-info.com/articulos/caracterizacion-de-la-carne-de-conejo

Rweyemamu, L. 2006. Challenges in the development of micronutrient rich food ingredients from soya beans Moringa oleifera.1-6 p. (en línea). Consultado 29 jul. 2011. Disponible en http://www.moringanews.org/doc/GB/Papers/Leonard_text_GB.pdf

Sun, B; Zhang, Y; Ding, M; Xil, Q; Liu, G; Li, Y; Liu, D; Chen, X. 2018. Effects of Moringa oleifera leaves as a substitute for alfalfa meal on nutrient digestibility, growth performance, carcass trait, meat quality, antioxidant capacity and biochemical parameters of rabbits. Animal Physiology and Animal Nutrition 102: 194-203. 